Wilfrid Laurier University

Scholars Commons @ Laurier

2008

\title{
First Principles Molecular Dynamics Study of CdS Nanostructure Temperature-Dependent Phase Stability
}

Bin Wen

Wilfrid Laurier University, bwen@wlu.ca

Roderick V.N. Melnik

Wilfrid Laurier University, rmelnik@wlu.ca

Follow this and additional works at: https://scholars.wlu.ca/math_faculty

\section{Recommended Citation}

Wen, Bin and Melnik, Roderick V.N., "First Principles Molecular Dynamics Study of CdS Nanostructure Temperature-Dependent Phase Stability" (2008). Mathematics Faculty Publications. 43.

https://scholars.wlu.ca/math_faculty/43

This Article is brought to you for free and open access by the Mathematics at Scholars Commons @ Laurier. It has been accepted for inclusion in Mathematics Faculty Publications by an authorized administrator of Scholars Commons@Laurier. For more information, please contact scholarscommons@wlu.ca. 


\title{
First principles molecular dynamics study of CdS nanostructure temperature-dependent phase stability
}

\author{
Bin Wen ${ }^{a)}$ and Roderick V. N. Melnik \\ $M^{2} N e T$ Lab, Wilfrid Laurier University, Waterloo, 75 University Ave. West, Ontario, Canada N2L 3C5
}

(Received 18 April 2008; accepted 6 June 2008; published online 1 July 2008)

\begin{abstract}
First principles molecular dynamics simulations are used to determine the relative stability of wurtzite, graphitic, and rocksalt phases of the $\mathrm{CdS}$ nanostructure at various temperatures. Our results indicate that in the temperature range from 300 to $450 \mathrm{~K}$, the phase stability sequence for the $\mathrm{CdS}$ nanostructure is rocksalt, wurtzite, and graphitic phases. The same situation holds for bulk $\mathrm{CdS}$ crystals under high pressure and $0 \mathrm{~K}$. Our work also demonstrates that although the temperature can affect the total energy of the CdS nanostructure, it cannot change its phase stability sequence in the temperature range studied in this letter. (C) 2008 American Institute of Physics.
\end{abstract}

[DOI: 10.1063/1.2952835]

Nanosized semiconductors have attracted much attention in recent years due to their unique optoelectronic properties and a wide range of current and potential applications. ${ }^{1-4}$ Among these semiconductors, cadmium sulfide (CdS) is one of the most important wide-gap semiconductors with good stability and high luminescence properties. ${ }^{5-7} \mathrm{CdS}$ nanostructures such as nanowires, nanorods, and nanodots have been grown experimentally, and they have been applied in photovoltaic cells, photonic switches, and optoelectronic devices. $^{2,8}$ In recent years, CdS nanostructures have also been used in biolabeling, bioimaging, drug delivery, and other biotechnological areas. ${ }^{5,9,10}$ In many of these optoelectronic and biorelated applications, CdS nanostructures have to operate in different temperature conditions, making the analysis of their thermal stability one of the most important avenues of research in this field. Additionally, CdS nanostructures such as quantum dots represent an intermediate state of matter between molecular species and bulk materials. ${ }^{5}$ Recently it has been shown that due to the large surface to volume ratio and reconstruction of surface crystal structure, the thermodynamic properties of the CdS nanostructure can differ significantly from bulk CdS. ${ }^{11}$ Nevertheless, the temperature-dependent phase stability of CdS nanostructures remains poorly understood, and systematic calculations and comprehensive comparisons of their thermodynamic stability under different temperature conditions are still lacking. Such calculations are essential for a better understanding of mechanical, electronic, and optical properties of these nanostructures. To fill this gap, in this work we carry out first principles molecular dynamics (MD) calculations for CdS nanostructures of various phases and study their temperature-dependent phase stability.

In this letter, we focus on the temperature-dependent phase stability of $\mathrm{CdS}$ nanostructures prepared for three different cases as follows. The initial structures are directly cut out from CdS wurtzite, graphitic, and rocksalt crystals, respectively. In particular, the structure in Figs 1(a)-1(c) is the wurtzite $\mathrm{CdS}$ nanostructure with a hexagonal prism structure, containing 96 atoms of eight layers (four $\mathrm{Cd}-\mathrm{S}$ double layers). The structure in Figs. 1(d)-1(f) is the graphitic CdS

\footnotetext{
a) Author to whom correspondence should be addressed. Electronic mail:
} bwen@wlu.ca.

nanostructure with a hexagonal prism structure, containing 96 atoms of four layers. Finally, the structure in Figs. $1(\mathrm{~g})-1(\mathrm{i})$ is the rocksalt $\mathrm{CdS}$ nanostructure, containing 96 atoms also. We denote them as 96A-WZ, 96A-GR, and 96A$\mathrm{RS}$, respectively.

The calculations described in this work were carried out by using two density functional theory packages: the CASTEP package $^{12}$ and the Car-Parrinello MD package (CPMD). ${ }^{13}$ The CASTEP package was used to calculatethe enthalpy of $\mathrm{CdS}$ under different pressures, while the CPMD package was used to calculate the total energy of $\mathrm{CdS}$ nanostructures under different temperatures. Our computational scheme based on the CASTEP package was designed in such a way that the interactions between the valence electrons and the ioncores were modeled by ultrasoft pseudopotentials. ${ }^{14}$ We used the generalized gradient approximation with the PBE exchangecorrelation functional. ${ }^{15}$ The $k$ point separation in the Brillouin zone of the reciprocal space was taken to be $0.04 \mathrm{~nm}^{-1}$,
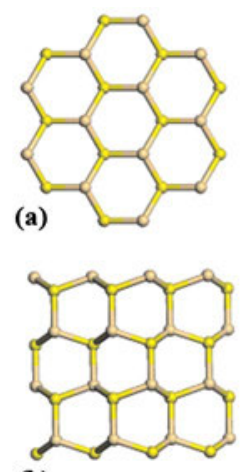

(b)

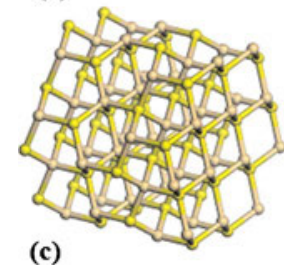

(c)

FIG. 1. (Color online) Side and top views of initial CdS nanostructures: (a) top view of 96A-WZ, (b) side view of 96A-WZ, (c) 3D view of 96A-WZ, (d) top view of 4L-96A-GR, (e) side view of 96A-GR, (f) 3D view of 96A-GR, (g) top view of 96A-RS, (h) side view of 96A-RS, and (i) 3D view of 96A-RS. 


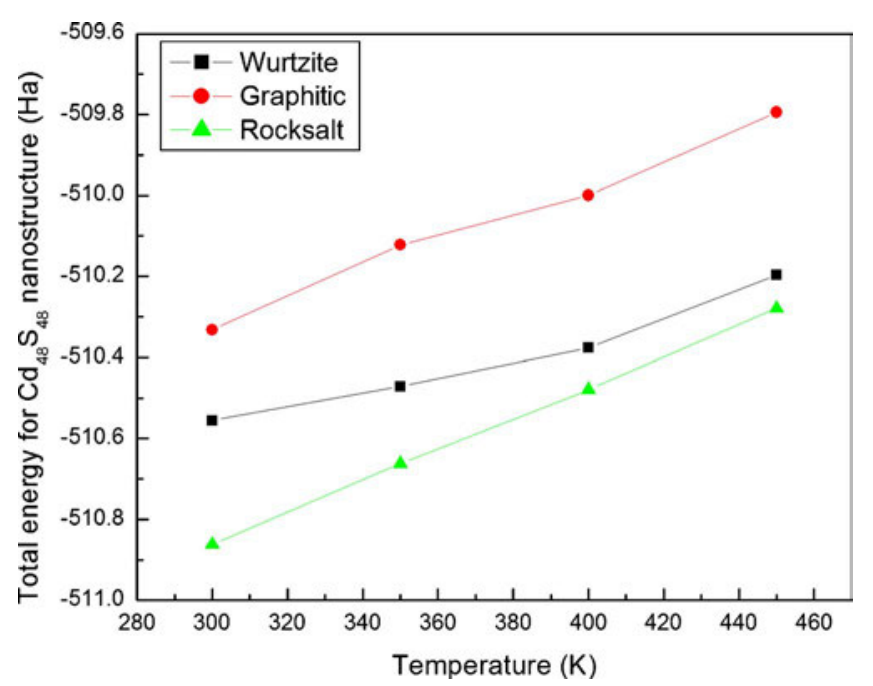

FIG. 2. (Color online) The relationship between total energy and temperature for various phases of the $\mathrm{Cd}_{48} \mathrm{~S}_{48}$ nanostructure.

which corrsponds to an $8 \times 8 \times 8 k$ point mesh. For the plane wave basis, we selected a high cutoff energy of $500 \mathrm{eV}$. Our computational scheme based on the CPMD package wasdesigned such that the interactions between the valence electrons and the ion cores were modeled by the HamannSchluter-Chiang norm-conserving methodology ${ }^{16}$ and by the Stumpf-Gonze-Scheffler (SGS) norm-conserving pseudopotentials ${ }^{17}$ for $\mathrm{Cd}$ and $\mathrm{S}$ elements, respectively. In details, the $\mathrm{Cd}$ pseudopotentials were generated from a $5 s^{1} 5 p^{0.25} 5 d^{0.25}$ reference configuration, and core radii $r_{c}$ with values of 1.6, 1.6, and 2.4 a.u. were used for $s, p$, and $d$ orbitals, respectively. The exchange-correlation effects were treated with local density approximation. ${ }^{18}$ The CPMD calculations were performed in a cubic box with $20 \AA$ sides periodic boundary conditions, and one point $(k=0)$ in the Brillouin zone. Test calculations for the vacuum region up to $15 \AA$ were performed, and they indicated that the interaction between nanostructures can be neglected when the vacuum region is larger than $6 \AA$. In our present work, the maximum dimension of the CdS nanostructure is about $13 \AA$, and the vacuum region between each nanostructure is about $7 \AA$. Our test calculations also indicated that the effect of the choice of $k$ point on the total energy is very small. The kinetic energy cutoff of the plane wave basis was set to $20 \mathrm{Ry}$. In this work, the time step for the Born-Oppenheimer MD is $0.12 \mathrm{fs}$ in the normal temperature and pressure ensemble, and the simulation time is 12 ps. Our simulation results indicated that simulation time of 12 fs is sufficient to equilibrate the nanostructure.

Based on the above two computational schemes, we first carried out trial calculations for a stress-free wurtzite $\mathrm{CdS}$ crystal. The calculated lattice parameters $a$ and $c$ were 4.13 and $6.78 \AA$ and 4.21 and $6.85 \AA$ for CPMD and CASTEP, respectively. These lattice parameters agree well with experimental values of 4.12 and $6.68 \AA{ }^{19}$ as well as with previous theoretical values of 4.11 and $6.63 \AA$. The $c: a$ ratios of 1.64 and 1.63 are also close to the ideal value of 1.63 for the hcp structure. Similar methodologies have already been applied to the analysis of several other nanostructures. ${ }^{20-22}$

Since the atom numbers are the same for different phases of the CdS nanostructure studied in this work, the relative stability of various phases of the CdS nanostructure under different temperature conditions can be determined by comparing the total energy. By performing first principles MD simulations, we calculated the total energy of these nanostructures under different temperatures, ranging from 300 to $450 \mathrm{~K}$. The relationships between the total energy and temperature for various phases of the $\mathrm{CdS}$ nanostructure are plotted in Fig. 2. From our calculations, the total energy $E_{t}$ increases almost linearly with temperature $T$ for all nanostructures studied in this work. This implies that for all CdS nanostructures studied here, stability decreases with an increase in temperature. For the rocksalt phase CdS nanostructure, with increasing temperature $T$ (in $\mathrm{K}$ ), the total energy $E_{t}^{\text {rocksalt }}$ (in hartrees) increases approximately according to a linear relationship: $E_{t}^{\text {rocksalt }}=-512.01952+0.003867$. For the wurtzite phase CdS nanostructure, this relationship can be expressed as $E_{t}^{\text {wurtzite }}=-511.27856+0.00234 T$, while for the graphitic phase CdS nanostructure, it is $E_{t}^{\text {graphitic }}=$ $-511.36445+0.00347 T$.
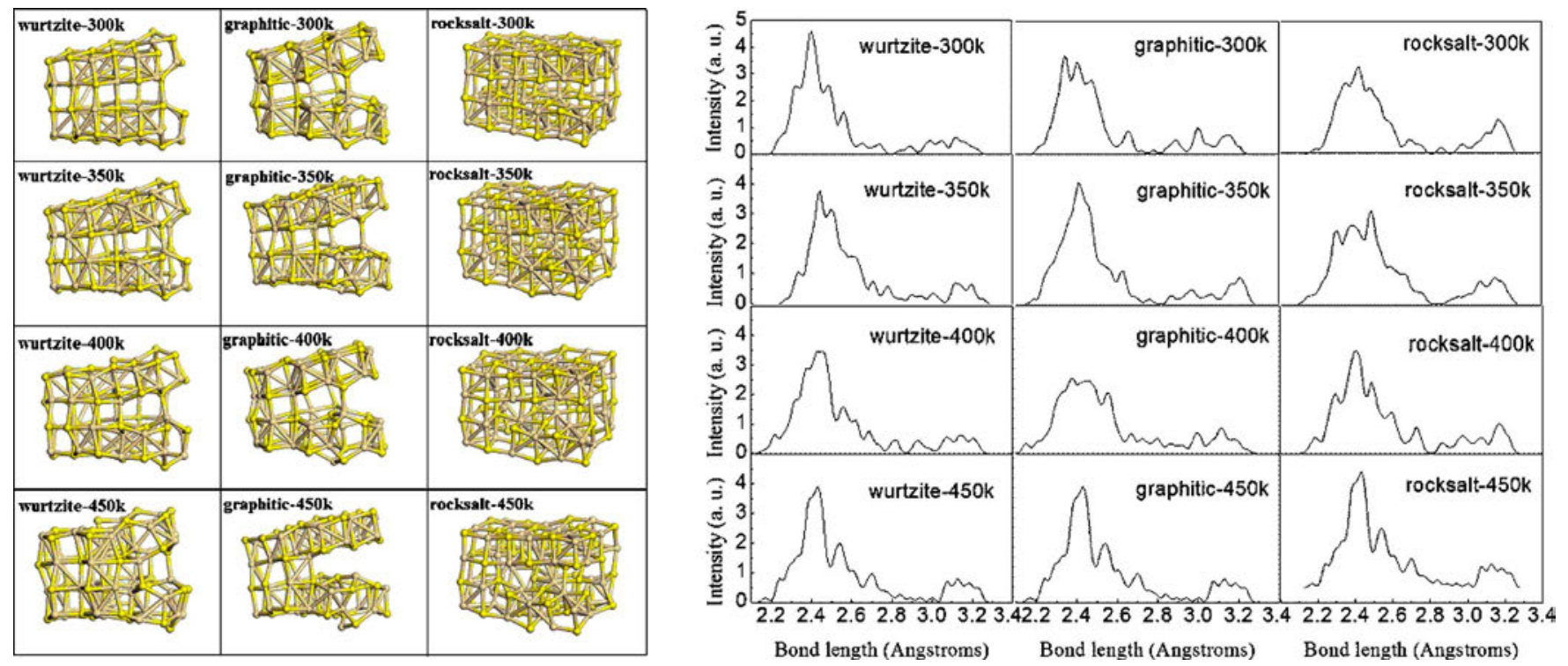

FIG. 3. (Color online) Snapshots (left) and bond length distribution (right) for various phase structures of nanosized $\mathrm{Cd}_{48} \mathrm{~S}_{48}$ after relaxation at different temperatures. 


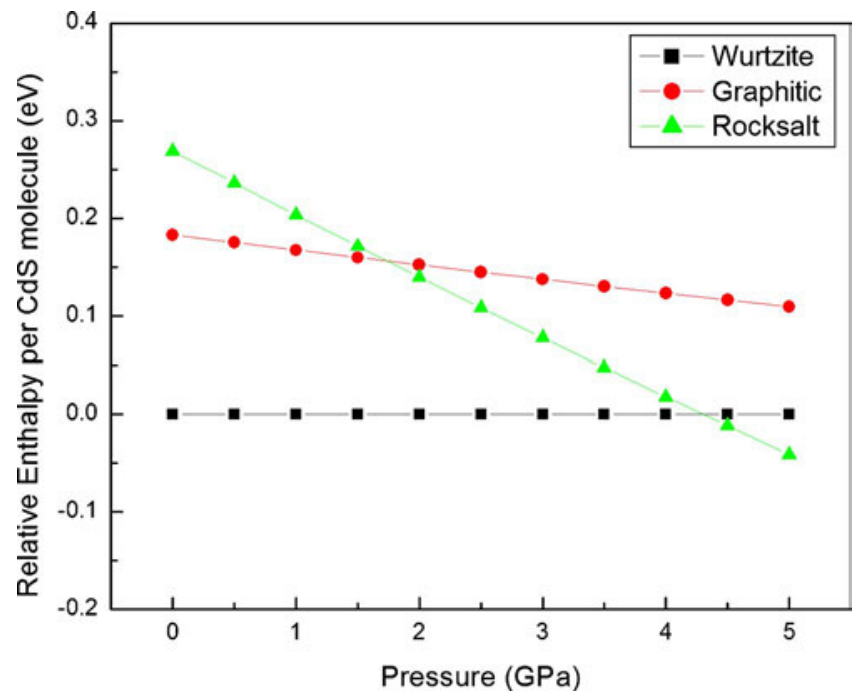

FIG. 4. (Color online) The relative enthalpy for wurtzite, graphitic, and rocksalt CdS crystals at pressure.

As can been seen in Fig. 2, there are no cross points for the total energy curves, which indicates that the stability sequence for the CdS nanostructures remains the same in the temperature range studied in this work. Furthermore, the stability sequence of these $\mathrm{CdS}$ nanostructures is rocksalt, wurtzite, and graphitic phases.

In order to understand the stability sequence of these nanostructures, their snapshots after relaxation at different temperatures and the corresponding bond distributions are shown in Fig. 3. With an increase in temperature, the number of broken bonds increases for all $\mathrm{CdS}$ nanostructures. At a temperature of $300 \mathrm{~K}$, only the rocksalt phase CdS nanostructure can maintain an initial input structure, while the wurtzite and graphitic phase $\mathrm{CdS}$ nanostructures cannot maintain it due to larger numbers of broken bonds. For the rocksalt $\mathrm{CdS}$ nanostructure, its crystal structure is maintained until the temperature of $400 \mathrm{~K}$ is reached, but the crystal structure is corrupted when the temperature increases further to $450 \mathrm{~K}$. Since the number of broken bonds increases with increasing temperature, the total energy also increases with an increase in temperature. The rocksalt $\mathrm{CdS}$ nanostructure can maintain its crystal structure under higher temperatures than the wurtzite and graphitic phase CdS nanostructures. This nanostructure is stable in the temperature range of $300-450 \mathrm{~K}$, in contrast to the other two CdS nanostructures.

It is well known that the wurtzite phase is stable for the bulk CdS at ambient conditions. However, in the above temperature range, as our calculation show, the rocksalt, a high pressure phase, is the most stable phase for CdS nanostructures. By using the Laplace-Yong equation for a spherical nanocrystal with diameter $x$, the internal pressure $P_{\text {in }}$, induced by the curvature, can be expressed as $P_{\text {in }}=4 f / x$, where $f$ denotes the surface stress. ${ }^{23}$ Thus, the total pressure is $P_{\text {tot }}=P_{\text {in }}+P$. If $P \approx 0$, then $P_{\text {tot }}=P_{\text {in }}=4 f / x$. For the nanosized materials, because the $x$ value is very small, the value of $P_{\text {in }}$ is very large and cannot be neglected. Therefore, the smaller the diameter $x$ is, the bigger the value of the totalpressure $P_{\text {tot }}$ becomes. In this work, the relationship between enthalpy and pressure for wurtzite, graphitic, and rocksalt CdS bulk crystals has also been calculated, and it is shown in Fig. 4. It can be seen that with an increase in pressure, the stability se- quence for wurtzite, graphitic, and rocksalt phases changes. At pressures ranging from 0 to $4.3 \mathrm{GPa}$, wurtzite is the stability phase. When the pressure is above $4.3 \mathrm{GPa}$, the rocksalt phase becomes the most stable, and the stability sequence in this case is rocksalt, wurtzite, and graphitic phases. The stability sequence for bulk CdS materials under high pressure is the same as that of CdS nanostructures at ambient conditions. These results imply that the curvature-induced internal pressure is a key reason for the phase stability of $\mathrm{CdS}$ nanostructures.

In summary, first principles MD simulations were carried out for wurtzite, graphitic, and rocksalt phases of the $\mathrm{Cd}_{48} \mathrm{~S}_{48}$ nanostructure, and the relationships between the total energy and temperature for different $\mathrm{CdS}$ nanostructures were deduced. Our computational results indicated that in the temperature range of $300-450 \mathrm{~K}$, the phase stability sequence for the $\mathrm{Cd}_{48} \mathrm{~S}_{48}$ nanostructure is rocksalt, wurtzite, and graphitic phass, which coincides with the stability sequence for bulk CdS crystals under high pressure. The temperature can noticeably affect the total energy of CdS nanostructures. However, it cannot change their phase stability sequence in the temperature range studied in this work.

This work, conducted in the $\mathrm{M}^{2} \mathrm{NeT}$ Laboratory (http:// www.m2netlab.wlu.ca), was made possible by the facilities of the SHARCNET. B.W. acknowledges computational support from Dalian University of Technology of China. R.M. acknowledges the support from the NSERC and CRC program.

${ }^{1}$ W. W. Yu, E. Chang, R. Drezek, and V. L. Colvin, Biochem. Biophys. Res. Commun. 348, 781 (2006).

${ }^{2}$ H. J. Lee, D. Y. Kim, J. S. Yoo, J. Bang, S. Kim, and S. M. Park, Bull. Korean Chem. Soc. 28, 953 (2007).

${ }^{3}$ R. Melnik and R. Mahapatra, Comput. Struct. 85, 698 (2007).

${ }^{4}$ R. Melnik and K. N. Zotsenko, Modell. Simul. Mater. Sci. Eng. 12, 465 (2004).

${ }^{5}$ A. Priyam, A. Chatterjee, S. C. Bhattacharya, and A. Saha, J. Cryst. Growth 340, 416 (2007).

${ }^{6}$ K. Barnham, J. L. Marques, J. Hassard, and P. O’Brien, Appl. Phys. Lett. 76, 1197 (2000).

${ }^{7}$ H. Cao, G. Wang, S. Zhang, X. Zhang, and D. Rabinovich, Inorg. Chem. 45, 5103 (2006).

${ }^{8}$ C. J. Barrelet, A. B. Greytak, and C. M. Lieber, Nano Lett. 4, 1981 (2004).

${ }^{9}$ X. Michalet, F. Pinaud, T. D. Lacoste, Ma. Dahan, M. P. Bruchez, A. P. Alivisatos, and S. Weiss, Single Mol. 2, 261 (2001).

${ }^{10}$ N. Gaponik, I. L. Radtchenko, G. B. Sukhorukov, and A. L. Rogach, Single Mol. 20, 1449 (2004).

${ }^{11}$ A. S. Barnard and H. Xu, J. Phys. Chem. C 111, 118112 (2007).

${ }^{12}$ M. D. Segall, P. J. D. Lindan, M. J. Probert, C. J. Pickard, P. J. Hasnip, S.

J. Clark, and M. C. Payne, J. Phys.: Condens. Matter 14, 2717 (2002).

${ }^{13}$ CPMD, Car-Parrinello Molecular Dynamics, V3.11.1, MPI für Festkörperforschung, Stuttgart.

${ }^{14}$ D. R. Hamann, M. Schluter, and C. Chiang, Phys. Rev. Lett. 43, 1494 (1979).

${ }^{15}$ P. Perdew, K. Burke, and M. Ernzerhof, Phys. Rev. Lett. 77, 3865 (1996).

${ }^{16}$ G. B. Bachelet, D. R. Hamann, and M. Schlter, Phys. Rev. B 26, 4199 (1982).

${ }^{17}$ X. Gonze, R. Stumpf, and M. Scheffler, Phys. Rev. B 44, 8503 (1991).

${ }^{18}$ P. Schrer, P. Krger, and J. Pollmann, Phys. Rev. B 47, 6971 (1993).

${ }^{19}$ J. Yang, J. Zeng, S. Yu, L. Yang, G. Zhou, and Y. Qian, Chem. Mater. 12, 3259 (2000).

${ }^{20}$ C. Li, W. Guo, Y. Kong, and H. Gao, Phys. Rev. B 76, 035322 (2007).

${ }^{21}$ C. Li, W. Guo, Y. Kong, and H. Gao, Appl. Phys. Lett. 90, 223102 (2007).

${ }^{22}$ W. Fan, H. Xu, A. L. Rosa, Th. Frauenheim, and R. Q. Zhang, Phys. Rev. B 76, 073302 (2007).

${ }^{23}$ R. C. Cammarata and K. Sieradazki, Annu. Rev. Mater. Sci. 24, 215 (1994). 